\title{
Project Portfolio Management for Academic Libraries: A Gentle Introduction
}

\section{Jennifer Vinopal}

In highly dynamic, service-oriented environments like academic libraries, much staff time is spent on initiatives to implement new products and services to meet users' evolving needs. Yet even in an environment where a sound project management process is applied, if we're not properly planning, managing, and controlling the organization's work in the aggregate, we will have difficulty achieving our strategic goals. Project portfolio management provides a way to ensure that this project work supports the organization's strategic vision, the active projects represent the highest priorities of the organization, and there are enough resources to accomplish all the project work at hand.

\section{Introduction}

\section{First, an Anecdote:}

During a plenary session at the Fall 2009 Digital Library Federation Forum, Sayeed Choudhury, the forum organizer, asked the roomful of more than sixty people for a show of hands in answer to a simple question: "How many of you finish projects at your institutions?" Only three or four hands went up. No one in the gathering of seasoned digital library technologists and project managers seemed surprised. Why not?

In highly dynamic, service-oriented, technology-rich work environments such as academic libraries, an increasing amount of staff time is spent on initiatives to design and implement new products and services to meet users' evolving needs. To be effective, organizations must be both nimble and efficient; they must spot trends and introduce new ser- vices while at the same time contending with budgetary constraints and limited resources. While some initiatives are conceived by upper-level management, service and workflow improvement projects are often initiated by the staff "in the trenches" who work most closely with users, see how they work, and hear their needs. While this makes for a lively and creative workplace, as formal and informal projects proliferate within an organization, staff can become overextended, taking on new initiatives in addition to the ongoing work that makes up the bread and butter of our library services. "Multitasking" staff can quickly feel overwhelmed with their work, and new projects, be they well or ill defined, tend to drag on and on for lack of clear prioritization and dedicated resources.

In addition to the need for sufficient resources, there are many reasons why

Jennifer Vinopal is Librarian for Digital Scholarship Initiatives in Bobst Library at New York University; e-mail: vinopal@nyu.edu. (C) 2012 Jennifer Vinopal, Attribution-NonCommercial (http://creativecommons. org/licenses/by-nc-sa/3.0/) CC BY-NC 
projects and initiatives may not be completed, including poor planning and oversight, ill-defined deliverables, and scope creep. These are the problems that project management was designed to prevent. Over the past decade, a growing number of articles in the library literature have recommended applying project management skills and processes to library work to better manage project planning and implementation and to thereby employ staff time in a more efficient way. The authors typically invoke the business world, especially IT and other service fields, to confirm project management's success in maintaining organizational efficiency. Winston and Hoffman point to Boeing, Horizon Blue Cross Blue Shield, and the U.S. Navy to explain, "As project management focuses on planning and the identification and the tracking of the use of human, technological and other resources, companies use project management to reduce the time needed for and cost of projects." 1

And, indeed, in applying project management methods, libraries have found efficiencies at the project level. However, to judge by the anecdote related at the beginning of this article as well as many informal conversations I have had with project managers working in academic libraries, this project-by-project management of time, money, and staff doesn't adequately resolve the organizational problem of project overload. "Projects in the multiproject environment share resources, and prioritization is not guided by any particular policy but rather by whoever seems to be screaming the loudest at any given time." ${ }^{2}$ Even in an environment where projects are well defined and where a project management process is appropriately applied, if we're not properly planning, managing, and controlling the organization's work in the aggregate, we will have difficulty achieving our strategic goals.

Project portfolio management (PPM) provides a method to mitigate this situation. In this article, I describe what PPM is and how libraries can benefit from it, and I provide examples of how PPM was introduced within New York University's Digital Library Technology Services (DLTS). I call it a "gentle introduction" for two reasons: 1) I hope that for its readers it is, in itself, a gentle introduction to PPM; 2) I advocate introducing PPM to your organization gently and applying only as much or as little as needed to accomplish your goals.

\section{What is Project Portfolio Management?}

First let's define some terms:

\section{What Is a Project?}

There are many definitions of "project" in the library literature and beyond. They all include the following common components: a project is an endeavor of limited duration, with a defined beginning and end, using specified and allocated resources (staff, money, equipment, and so on) to accomplish a specific objective. Organizations will tailor this definition to their own needs and situations (for example, some companies might also stipulate that only endeavors that last more than " $\mathrm{N}$ " number of weeks or use " $X$ " number of staff hours will be considered a project). Project management is the process by which an individual project is organized, overseen, and administered throughout its duration.

\section{What Is a Project Portfolio?}

A project portfolio (or project registry) is a list or inventory of all the present and future projects of the department, organization, or institution being overseen. (In this article, I use the generic term "organization" to mean any unit or part of an institution that is seeking to benefit from PPM). The portfolio should be comprehensive and will thus include all initiatives that meet the organization's definition of "project." As a result, it will likely reveal hidden work that had previously either been overlooked by management or had not been properly 
classified as a project. The portfolio will also contain certain standard information or data about each project, so projects can be compared with each other and data analysis can be done across the portfolio in the present and over time. How much or little information is gathered depends on the organization and its needs, but the portfolio typically includes for each project: scope/charter statement, start and end dates, staffing (resource allocation), budget, and an indication of how the project aligns with the organization's strategic goals (strategic alignment). It may also include deliverables and milestones, as well as information about major changes to the projects as they proceed (for instance, changes in end dates). With the broad overview this inventory provides, those managing the portfolio can better review and consider the scope of the work being done within the organization and can then think strategically about the organization's work in ways that would be impossible having only a project-byproject view.

\section{What Is Project Portfolio Management?}

PPM is an ongoing process by which management can ensure, in an organized and ongoing way, that: 1) the project work of the organization supports the strategic vision and directions of the organization; 2) the set of active projects represents the highest priorities of the organization; 3) there are enough resources available to accomplish all the project work at hand; and 4) there are procedures that can be enacted to correct course when problems are discovered in portfolio alignment with strategic vision, prioritization, or resource allocation. The process of PPM includes regular meetings of the group tasked with managing the portfolio to review the portfolio and to accomplish the goals listed above. Whether the PPM process is extensive (as it is in large corporations) or modest, the goals of PPM are the same: to prioritize work (for strategic alignment); to manage resources (staff, money, time, and so forth); and to manage risk (of failure, cost overruns, and the like). Just as the goal of project management is to increase productivity and effectiveness at the project level, project portfolio management is designed to increase efficiency at the organizational level.

\section{Who Oversees the Portfolio?}

The governance of the portfolio depends on the needs of the organization and the scope of the portfolio. Large corporations usually have a Project Office or Project Management Office (PO or PMO) whose job it is to maintain and manage the project portfolio, as well as to ensure that project management is practiced appropriately throughout the organization. In smaller organizations, there may be just one staff member or a portion of an FTE tasked with implementing or leading the PPM process. The portfolio may be managed or governed by an individual or a management group. Those who govern the portfolio should be able to maintain a strategic perspective on the portfolio in relation to the organization's goals and should have the authority to make or advocate for changes in the portfolio's makeup. In any organization, large or small, for PPM to be successful it is crucial to have the understanding, buy-in, and cooperation of all the staff who will be participating in its application. I further address organizational culture and PPM below.

\section{The Benefits of PPM}

PPM is making its way from the business world into higher education mainly via campus IT units, and there have been some recent articles and presentations on PPM use in this academic setting. ${ }^{3}$ Based on conversations I've had at professional meetings, I know that some digital library teams are also exploring PPM's benefits. But, despite the clear benefits it can provide to a mixed or even non-IT portfolio, PPM hasn't yet gained a firm foothold in general library operations. In my literature review, I found no outright references to PPM in the library literature 
and only two brief references to strategies for managing multiple projects, which is more akin to what is now called program management than it is to portfolio management. ${ }^{4}$

Books and articles about PPM in the business world typically describe these benefits: strategic alignment and project prioritization, reduced waste and cost savings, performance assessment, ability to forecast resource needs, risk balancing, and early warnings before problems spread across the portfolio. ${ }^{5}$ The $\mathrm{CIO}$ for the University of Nebraska notes that a PPM process that is well-tuned to the needs and character of the organization can also create a more professional atmosphere for analysis and communication: “Using portfolio management techniques, we can move from the subjective and sometimes emotional debates about whose project we do first to engage in more objective and informed discussions about how to use our limited resources to best serve the university." 6 The resulting gains in organizational efficiency and emphasis on proactive planning can also increase staff satisfaction: "by bringing the organizational workload under control and eliminating the need for constant fire fighting, the project office can positively affect individual - and corporate-well-being." ${ }^{\prime 7}$

PPM is a continual or iterative process that allows organizations to observe what is happening in the present and to analyze and learn from the past to better plan for the future. Rather than thinking about projects as work silos, when we review the project inventory we look holistically at the portfolio and are very attentive to relationships and interdependencies among initiatives (such as common resources, deliverables, or workflows). For example, the portfolio management team might notice that three distinct projects are developing similar workflows or tools to solve three separate problems. From a portfolio perspective, that deliverable (the service in question) has the potential to provide the organization greater value and efficiency if it is designed in a way to be reused across multiple projects (both current and future). So the management team may decide to evaluate the impact this service could have across the entire portfolio and request that those three projects or deliverables be collapsed into one, with the requirements being revised to meet a broader set of needs. Or the management team may decide to make two of the projects dependent on the third and stage them sequentially so that the later projects can simply use the service developed in the first rather than create their own.

The broad overview and collection of information that the portfolio provides allows organizations to move from gut feelings and anecdotal evidence to solid observations based on data. In the portfolio, it is easy to find answers to questions like: "How often do our projects end late?" "At what point over the coming year will our workload lighten enough for us to take on new projects?" and "Which staff are overcommitted on projects?" Over time, as these data accumulate, the portfolio manager(s) will be able to ask deeper questions and observe trends. Through such informed review, portfolio managers may also institute organizationwide solutions to problems that individual project managers, with their more restricted field of view, would rarely consider. For example, as we track modifications to project end dates and the reasons for these changes, we might learn that our projects frequently end late because of scope creep, because project managers are not estimating work duration well, or because staff are overcommitted and are unable to complete their work on schedule as other projects compete for their time. These observations can in turn lead to solutions like: providing project managers with additional training so they can more effectively plan and manage projects, rescheduling projects, rebalancing or shrinking the portfolio, or deciding to outsource some project work so in-house staff can better focus their at- 
tention on getting their tasks and projects done on time. The same is true for gaps in skill sets. Instead of rejecting a proposed project because the organization doesn't have the staff or skill, the management team might instead decide that the project is important enough to the organization and the portfolio that they will train staff, or add this skill through outsourcing, hiring temporarily, or hiring permanently. When used in this way as a performance assessment tool, the data in the portfolio might even inform high-level organizational or institutional initiatives such as reorganization and strategic planning.

In the next section, I provide suggestions for introducing PPM into an academic library setting and give examples from the ongoing implementation of PPM in NYU's DLTS. Although NYU's DLTS serves as a case study, the goal of this article is to encourage the use of PPM in any organization seeking to benefit from a more systematic approach to coordinating and prioritizing project work. While campus IT and some digital library units have been early adopters of PPM, the advantages of PPM are not specific to IT work. In fact, the routine use of PPM for all library projects - be they related to reference service development, building renovation, cataloging, strategic planning, or software development-would provide a more consistent and dependable approach to evaluating, prioritizing, and staffing initiatives across the entire library.

\section{Introducing the Project Portfolio Management Process into an Academic Library Setting Organizational Change}

Establishing portfolio management in an organization requires a change in culture to incorporate more reflection, analysis, and planning into the organization's activities. According to Stephen Bonham, one of the keys to the successful rollout of a new project management office (PMO) is for upper-level management to endorse PPM as an important strategic initiative: "Because there will be conflict when rolling out the PMO, clear and prompt executive support is mandatory. It will need to be made crystal clear to the troops that the PMO is central to the success of the company." ${ }^{8}$ However, in an academic environment where we don't think of staff as obedient "troops," it's not enough for leadership to prescribe better efficiency and resource management. PPM takes staff time and effort to implement; for this reason, successful organizational adoption is not guaranteed. To succeed, staff as well as management need to understand and see the benefits of PPM and be willing to participate in the process, which may include greater information tracking and planning than we are used to. However, just as planning, assessment, and accountability are not antithetical to having an innovative culture, PPM doesn't have to be constricting or represent a "corporatization" of the academic library. Rather, by providing a method for analyzing and prioritizing work, the PPM process can help organizations free up the time and resources to strategically focus on the work they value most.

\section{Understand Your Needs and Streamline Your Implementation}

Project portfolio management is a set of methods that should be customized to meet the goals of the organization. Defining the key deliverables of the PPM process at the outset will significantly reduce the risk of overinvesting in unproductive processes and data collection. In his presentation on developing a PPM process for IT Governance at Franklin University, Patrick Bennett describes how they adapted a corporate PPM model to meet their local needs. ${ }^{9}$ Before designing a process, it is crucial to understand the issues or problems that PPM is intended to address within the organization. Regarding the implementation of PPM at the University of Nebraska, the CIO explains "the goal is to get a good handle on understanding the needs of the intended audience with as much specificity as possible. What decisions are they going to 
be asked to make? How can the data be arranged in a manner that makes the pertinent issues visible? These requirements are critical and will focus effort during the remaining phases of the [PPM implementation] project."10 The U.K. Office of Government Commerce, which created a maturity framework called P3M3 to rate an organization's portfolio management maturity, states: “The five-level hierarchy of P3M3 does not imply that every organization should aim for, or needs to achieve, Level 5 in all three sub-models [portfolio management, program management, and project management]. Each organization should decide which Maturity Level would be optimal for its particular business needs at a given time."11

In the summer of 2009, NYU's DLTS began considering PPM as a way to better organize and plan the department's work. The number of projects and services we supported was growing, deadlines were frequently overrun because staff were overcommitted on projects, and we had no clear agreement on what our priorities were. DLTS staff as well as leadership were ready for a change, so I was asked to design and introduce a PPM process for the team. It was clear from the start that a wholesale application of PPM as it is practiced in the business world was out of the question. In large organizations with well-developed project management environments, the project portfolio includes an extensive set of data: "It requires collecting the most fundamental data about work, including project names, start and end dates, the names of people performing the work, and how many hours each person charged to the effort. All of this must be associated with individual payroll records, and operating costs must be applied as overhead." ${ }^{12}$ This level of data collection and tracking was too extensive and unnecessary for our needs. Instead, we opted for a more modest, customizable process that wouldn't burden already busy staff and that would be easy for me to set up and manage by devoting just a few hours a week of my time.
As I learned more and more about PPM in the business world and thought about our organizational culture, I developed some requirements for our implementation: we would develop the easiest, least time-consuming process to accomplish our goals; there would be no methodology or documentation for its own sake; this would be a shared process with a shared toolset and a shared product; whatever tools we adopted must be easy to set up and use and must facilitate data sharing. To build momentum with this new process, it was important to have active portfolio oversight and management right from the start. Rather than creating a whole new management infrastructure to support PPM, it can be more efficient for organizations to assign the portfolio management responsibility to an existing group for which this activity would be a strategic goal. This could be, for example, the library's department managers group, an existing departmental leadership team (such as a technical services or public services management team), or a high-level working group that cuts across the organization (like an organizational development working group). At NYU's DLTS we decided that our existing Digital Library Management Team (a six-person group composed of the DLTS director plus five DLTS staff with management responsibilities) would act as the project governance group and would help me design and test the PPM process. This collaborative approach helped us come to early consensus on which aspects we would and wouldn't focus on and made our PPM as responsive as possible to our needs. Since this process was new and experimental for us, to prevent disruption to the department's work we decided that, initially, management alone would shoulder the burden of this initiative; nonmanagement staff would be updated on the implementation and would benefit from its success, but they would not be directly involved in the PPM work itself. 


\section{Make PPM a Learning Process}

We took an incremental approach to implementing PPM, introducing new features as needed and regularly assessing what we had done to determine if the payoff was worth our effort, then revising as necessary. "The incremental approach provides the time necessary for this process of organizational learning to take place between consecutive deployment increments.... [A]n incremental approach prevents the common tendency to overengineer technology solutions while substantially shortening the time to the arrival of business benefits." ${ }^{\prime 13}$ The goal was to avoid unnecessary work and to achieve quick wins so we would all feel the benefits of PPM right away.

The first and, surprisingly, most timeconsuming task was to create our project inventory. The project inventory is not only the foundation of the PPM process, but it is a great way to expose hidden work and give staff public credit for the work that they are doing. Over a period of months I interviewed the other members of the management group to learn what work they and their staffs were doing, what they wanted to get out of the PPM process, and what kinds of project information they would like to track over time. As we reviewed and cataloged our project work, one unexpected area of disagreement was which of our activities were actually projects and how to distinguish between a project and a service. Historically, DLTS's work had been primarily project based, and we were in the habit of calling all of our work "projects." This was unwise because, by confusing projects (which have a defined end date) with services (which are ongoing), we were internalizing the mistaken idea that projects never end; and, except for the grant-funded projects, we were not feeling the imperative to complete them. We realized that we needed not only to create working definitions for "project" and "service," but also to emphasize the importance of proper project methodologies (for example, defined deliverables and end dates) in project initiation and during project review in our regular management meetings. (A side benefit of this "project vs. service" conversation was the beginning of what is now our service portfolio.)

As you can see, project management and portfolio management are tightly linked; the ability to do the latter depends on the former. The assessment, predictions, and decisions you base on the portfolio are only as good as the project information therein. This is why, in the corporate world or in other large organizations, in addition to managing the project portfolio, a large project management office may be responsible for developing and promoting good project management practices throughout the organization (including hiring, training, and overseeing the project managers themselves). But, even on a much smaller scale, portfolio management can be a driver for better adoption and application of project management within the organization. As the PPM implementation proceeds, the portfolio management team's need for more accurate information will create pressure to improve the project management process. Since the members of the NYU DLTS management team are also typically the project managers of the department's projects, throughout the PPM implementation we have all become more sensitive to the fact that decisions or changes at the project level have portfolio-level implications. And, as the "project vs. service" anecdote above illustrates, although our project management practice may sometimes be quite informal, this dependent relationship between PPM and PM inspired us to require for larger projects some basic project methodology such as project charters stating requirements and nonrequirements (to combat scope creep), project timelines, realistic end dates, and which staff were responsible for which requirements during what time frames.

\section{Implementing PPM at NYU's Digital Library Technology Services}

In this final section, I relate some of the 
practical steps we took since summer 2009 toward implementing PPM at NYU's DLTS and I describe our current process and goals. My responsibilities managing the PPM process represent a small portion of my work at NYU Libraries. I knew that, with just a few hours per week to spare on PPM, the initiation of this process would take some time. So I designed the planning period to have a minimal impact on others' work until I could assemble a large enough body of knowledge that we could effectively act upon. I devoted the first six months of our implementation in 2009 to learning about PPM, interviewing the other management team members about their goals for the process, gathering project information to populate the portfolio, and evaluating potential tools. Enterprise-level project management/ PPM systems, which enable comprehensive documentation and analysis (such as Microsoft Project Server), were far too expensive and complex; they seemed, frankly, too "corporate" for our needs. (Although NYU ITS uses AtTask for project and portfolio management, in DLTS we chose to keep our PPM process separate and tailored specifically to our own needs.) And the free or low-cost online project management and collaboration tools I reviewed all fell short for one reason or another. I instead chose a tool everyone was already familiar with, Google spreadsheets, which is free, easy to use, designed for group access and editing, and is customizable enough for our current needs. We track basic information for each project: project name, start and end dates, brief description, project manager, status, priority, and notes. (We also have a column called "project grouping," which I describe below.) We have one spreadsheet for all "Current/Potential" projects (that is, anything with a status of "active," "on deck," or "requested"), a second sheet for "Completed" projects, and a third for "Cancelled/No-Low Priority." We group "active" projects with "on deck" and "requested" projects to give us a broad view of all proposed initiatives so we can have a sense of our prospective workload and to make it easier for us to review, prioritize, and look for relationships among these projects. A Google gadget linked to the "Current/Potential" projects sheet plots each project's duration against a running calendar (see figure 1). This simple visualization allows us to easily see when the projects in the portfolio are scheduled to end and helps us predict when we will be able to take on new projects.

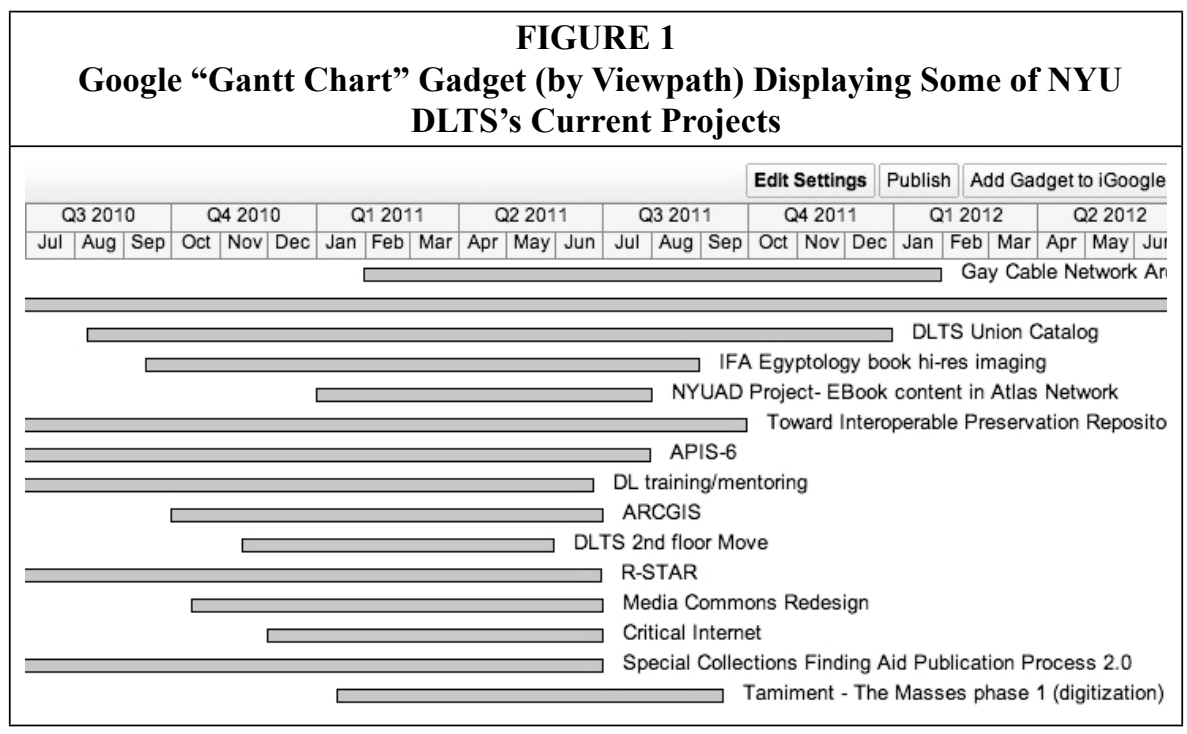


As the portfolio manager, my role is to keep the management team actively thinking about the portfolio and to advocate for any methodological changes that will improve our ability to manage our workload. One of our key goals in adopting PPM was to develop a shared prioritization process that would help us think more strategically about work assignments and would give staff a way to choose among competing demands. To create a common language for discussing priorities and making decisions, I developed a set of terms with definitions that take into account the kinds of organizational and institutional concerns that typically influence our decision making:

1. None: not an organizational or institutional priority;

2. Low: low priority; it would be nice if we did it, but there's no organizational or institutional mandate;

3. Normal: we've made a commitment to this project but no other projects are dependent on its timeline; it can be deferred in favor of other, higher-priority projects;

4. High: we've made a commitment to this project; other projects are dependent on its timeline; other factors require timely completion (such as grant funded, VIP attention); project is on track and will remain so if current conditions continue;

5. Urgent: high-priority project at risk of failure; needs immediate attention; other high-priority projects are dependent on its timeline; other factors require timely completion (such as grant funded, VIP attention).

Every active project in the portfolio must, at the very least, be assigned start and end dates, a project manager, and a priority. For larger projects, we are also working to create charters that include a project description, team members with assignments, requirements and nonrequirements, and project timelines. We review the portfolio briefly at our weekly Digital Library Management Group meeting. During this time, we may discuss the status of projects and consider problems (especially those that will affect other projects); assign or revise priorities; allocate resources; and evaluate potential projects. As we work, we project the spreadsheet onto a screen and edit it in real time so we come to agreement on changes and document all decisions before the close of the meeting. For more in-depth review, we schedule longer working meetings as needed.

During a recent "portfolio management retreat," we discussed our team goals and how we might adapt our portfolio management process to help us achieve them. We agreed that our objective in doing projects should be to build sustainable, reusable tools and services and that the next phase of PPM should focus on facilitating this goal. As a first step, we have begun to assign all active, on-deck, and requested projects a "project grouping" designation (imaging, book publication, video processing, preservation strategy, and the like) that enables us to sort the portfolio according to these parameters. Although the approach may sound simplistic, with 80-100 projects typically on this list, we were previously unable to get a quick snapshot of potentially related initiatives. With this simple device, we can see commonalities across the portfolio that we hadn't noticed before. Now, as we initiate new projects, we try to group similar projects or subprojects together to build tools and services once that will satisfy multiple needs now and into the future. As a result, we will modify our prioritization process to give more weight to projects that allow us to group deliverables from several projects. The payoff of this grouped approach includes more productive use of staff time as we accomplish more work through a single initiative, as well as greater future value in the form of robust, reusable services. 
We are also starting to assemble a service portfolio, similar to the project portfolio, so we can have a more complete picture of team activities and staff effort across all of our department's initiatives.

\section{Conclusion}

At the 2009 Digital Library Federation Forum mentioned in the anecdote at the beginning of this article, I attended the half-day Project Managers Group meeting at which we discussed obstacles to innovation in academic libraries. Impediments that the group identified included: lack of time and resources; the lack of institutional will to prioritize; no clear process to transition projects into production services; and continued support for outdated, legacy services. These same organizational challenges cause project (and service) overload, deferred deadlines, staff overcommitment, and eventually negative morale. While portfolio management isn't a cure-all for every organizational ill, for the institution seeking ways to address these cultural and management problems, PPM does provide a structure, the data, and a process to: acknowledge, through the project inventory, the full extent of the organization's current project work; evaluate, prioritize, and deprioritize projects against the goals of the organization; assess performance; and encourage the application of sound project management practices.

At NYU's DLTS, our PPM process isn't perfect. We are still learning to better estimate project duration so our portfolio projections are more accurate. The kind of project reporting we can produce from Google spreadsheets is minimal, and we may eventually need to move to a more sophisticated portfolio tool. The best-laid project prioritization plans and timelines can be interrupted by an unexpected VIP request or a high-priority problem. And we haven't committed to tracking staff effort hours across the project and service portfolios, without which we can't reliably allocate people to initiatives. Based on the U.K. Governments P3M3 management maturity framework, with level five being the highest level of implementation, I would rate our departmental PPM adoption between a level one ("awareness of process") and two ("repeatable process"). ${ }^{14}$ But whatever our maturity level, PPM has helped us begin to create what Patrick Bennett calls a "project-minded culture" that has a transparent, rational, and shared process for communicating about, planning, and accomplishing our work. ${ }^{15}$ We talk more openly about workloads and come to agreement about priorities. No one's work is invisible because our initiatives are documented for all to see. We rationally discuss our ability to undertake new work and back up our opinions with data. And we continue to explore new ways to keep our work strategic, efficient, and rewarding. It's not perfect, but perhaps that's good enough.

\section{Notes}

1. Mark D. Winston and Tara Hoffman, "Project Management in Libraries," Journal of Library Administration 42, no. 1 (2005): 53.

2. Eduardo Miranda, Running the Successful Hi-Tech Project Office (Norwood, Mass.: Artech House, 2003), 2.

3. Marketwire, "Higher Education IT Project and Portfolio Management Focus of First Annual TeamDynamixHE F2F User Group Meeting," The Street (Mar. 15, 2011), available online at http://t. co/lylvwwa [accessed 4 April 2011]; Lawrence Lim and Aileen Koh, "IT Portfolio Management in Higher Education" (presentation, Australian Institute of Project Management, Adelaide, Australia, 2009), available online at http://epublications.bond.edu.au/library_pubs/22 [accessed 4 April 2011]; Patrick A. Bennett, "Project Portfolio Management in Higher Education: It's Not All about the Money" (presentation, Educause Midwest Regional Conference 2009, Chicago, Ill., 2009), available online at http://www.educause.edu/Resources/ProjectPortfolioManagementinHi/169314 [accessed 4 April 2011]; Walter Weir, IT Portfolio Management and Accountability in Higher Education, Research Bulletin (Washington, D.C.: Educause Center for Applied Research, 2006), available 
online at http://net.educause.edu/ir/library/pdf/ERB0614.pdf [accessed 4 April 2011].

4. Barbara Allan, Project Management: Tools and Techniques for Today's ILS Professional (London: Facet Publishing, 2004), 122; Liz MacLachlan, Making Project Management Work for You (London: Library Association, 1996), 75.

5. Bryan Maizlish and Robert Handler, IT Portfolio Management Step-by-Step: Unlocking the Business Value of Technology (Hoboken, N.J.: John Wiley \& Sons, 2005), Introduction, available online at http://common.books24x7.com/book/id_16906/book.aspx [accessed 14 April 2011]; Miranda, Running the Successful Hi-Tech Project Office, 58-59.

6. Weir, IT Portfolio Management and Accountability in Higher Education, 3.

7. Miranda, Running the Successful Hi-Tech Project Office, 9.

8. Stephen Bonham, IT Project Portfolio Management (Norwood, Mass.: Artech House, 2004), 106.

9. Bennett, "Project Portfolio Management in Higher Education," 13.

10. Weir, IT Portfolio Management and Accountability in Higher Education, 5.

11. Rod Sowden, David Hinley, and Steve Clarke, Portfolio, Programme and Project Management Maturity Model; P3M3 Public Consultation Draft v. 2.0 (London: Office of Government Commerce, 2008), 18, available online at www.ogc.gov.uk/documents/p3m3.pdf [accessed 12 May 2011].

12. Weir, IT Portfolio Management and Accountability in Higher Education, 5.

13. Miranda, Running the Successful Hi-Tech Project Office, 215. 10.

14. Sowden, Hinley, and Clarke, Portfolio, Programme and Project Management Maturity Model,

15. Bennett, "Project Portfolio Management in Higher Education," 29. 